\title{
What is the optimum duration of anticoagulation for the management of patients with idiopathic deep venous thrombosis and pulmonary embolism?
}

\author{
${ }^{1}$ TM Hyers, ${ }^{2} \mathrm{HG}$ Shetty, ${ }^{3} \mathrm{IA}$ Campbell \\ ${ }^{1}$ Clinical Professor of Internal Medicine, St Louis University School of Medicine, St Louis, Missouri, USA; ${ }^{2}$ Consultant Physician and \\ Honorary Senior Lecturer, Univerity Hospital of Wales and Cardiff University Medical School, Cardiff; ${ }^{3}$ Honorary Consultant Physician, \\ Llandough Hospital, Llandough, UK
}

The optimum duration of anticoagulation following idiopathic deep venous thrombosis and pulmonary embolism is controversial. The JRCPE is delighted to publish a debate written by three international experts. Dr Shetty and Dr Campbell recommend that three months' anticoagulation is sufficient, while Professor Hyers recommends anticoagulation for longer than three months.

KEYWORDS Anticoagulant therapy, idiopathic venous thromboembolism DECLARATION OF INTERESTS No conflict of interests declared.

\author{
Correspondence to \\ TM Hyers \\ 522 North New Ballas Road, Suite \\ 350, Saint Louis, MO, 63I4I, USA \\ tel. +l 3146999383 \\ e-mail thyers@careinternet.com \\ HG Shetty \\ C6 Link, University Hospital of \\ Wales, Cardiff CFI4 4XW, UK \\ tel. +44 (0)29 $20744967 / 3867$ \\ e-mail hamsaraj.shetty@wales.nhs.uk
}

\section{Three months of anticoagulation is sufficient}

\author{
HG Shetty, IA Campbell
}

\begin{abstract}
Anticoagulant therapy is highly effective in preventing recurrent events in patients with venous thromboembolism (VTE). For patients with 'secondary' or 'provoked'VTE affecting isolated calf veins or upper limb, there is general agreement that six weeks to three months of anticoagulation is adequate. Those with 'provoked' iliofemoral deep vein thrombosis (DVT) or pulmonary embolism (PE) are usually treated for three or six months. The duration of treatment in patients with idiopathic VTE, however, is controversial. Some experts recommend long-term anticoagulation after the first episode of VTE, whereas others, including ourselves, advocate only three months of anticoagulation. ${ }^{1,2}$ Although a recurrent event such as a massive PE can be fatal, haemorrhagic complications of anticoagulation can be equally dangerous. Therefore, a decision to continue anticoagulant therapy beyond three months should not be taken lightly. We believe that long-term treatment should not be considered universally for all patients with idiopathic VTE. For our contribution to this debate we have reviewed the literature and presented the evidence supporting our view.
\end{abstract}

\section{THE HYPOTHESIS}

Proponents of long-term anticoagulant therapy are assuming that every person who has had an idiopathic DVT or PE will have an ongoing thrombophilic tendency and therefore is highly likely to develop recurrent events once anticoagulation is discontinued. This is unlikely to be the case as the aetiology of idiopathic VTE is likely to be diverse and the risk for recurrence is also likely to be variable. Indiscriminate long-term anticoagulant treatment in every such person is clearly not a logical option.

\section{RISK OF RECURRENT EVENTS}

We randomised 369 patients with proximal DVT or PE ( $42 \%$ idiopathic) for three months and 380 patients ( $47 \%$ idiopathic) for six months of anticoagulant therapy. Fatal and non-fatal failures of resolution, extension or recurrence during and after three or six months of anticoagulation were similar between the two groups, at $8 \%$ each. Major haemorrhages during treatment occurred in $2 \%$ of the six months group and in none of the patients randomised to the three months group. ${ }^{3}$

A prospective cohort study has reported that the risk of any fatal PE and definite or probable fatal PE after discontinuation of anticoagulation is 0.49 events ( $95 \%$ confidence interval, $\mathrm{Cl}, 0.36-0.64$ events) per 100 person-years and 0.19 events $(\mathrm{Cl} 0.12-0.30$ events) per 100 person-years, respectively, and that the case-fatality rate for recurrence is $9.0 \%(\mathrm{Cl} 6.8-\mathrm{II} .8 \%)$ for any fatal $\mathrm{PE}$ and $3.8 \%(\mathrm{Cl} 2.4-5.9 \%)$ for definite or probable fatal $\mathrm{PE}$ in patients with a first episode of symptomatic VTE who received anticoagulant therapy for a mean duration 
of six months (range 3-39 months). In this study, following the first episode of symptomatic VTE, the annual risk of definite or probable fatal PE was 0.15 (Cl 0.08-0.26) per 100 person-years after the first year. Only I2/I, I 38 patients with idiopathic VTE had definite or probable fatal PE after 4,844 person-years' follow-up. ${ }^{4}$

Another study randomised patients with a first episode of idiopathic proximal deep venous thrombosis who had completed three months of oral anticoagulant therapy to either discontinue oral anticoagulants or to continue for nine additional months. Both groups were followed up for an average of over 37 months. Primary intentionto-treat analysis showed that $21 / 134$ (15.7\%) patients assigned to continued oral anticoagulant therapy had a recurrent VTE, as compared with $2 \mathrm{I} / \mathrm{I} 33$ (I5.8\%) patients who discontinued anticoagulant therapy (relative risk: 0.99; $95 \% \mathrm{Cl}, 0.57-1.73$ ). The incidence of recurrence after the discontinuation of treatment was $5.1 \%$ per patient-year in patients in the three months group and $5.0 \%$ per patient-year in the extended treatment group. None of the recurrent VTEs was fatal. Non-fatal major bleeding occurred in $1.5 \%$ of patients in the three months group and in $3 \%$ of patients in the extended period group. ${ }^{5}$ There were no fatalities due to bleeding in the extended treatment group. Thus, prolonged anticoagulation did not reduce the risk of recurrent VTE but did increase the risk of major bleeding.

\section{RISK OF BLEEDING}

One of the major considerations in prescribing anticoagulants is the risk of bleeding associated with their use. A meta-analysis, aimed at estimating the clinical impact of anticoagulant-related bleeding, included 33 randomised controlled trials and prospective cohort studies which investigated patients with VTE, and reported major bleeding and death as primary study outcomes. The patients received 4,374 patient-years of oral anticoagulant therapy (target international normalised ratio, INR, 2.0-3.0) for at least three months. The metaanalysis found that around one in seven bleeding episodes with oral anticoagulant therapy is fatal or intracranial. ${ }^{6}$ The overall case fatality was around $13.4 \%$, with a major bleeding and intracranial bleeding rate of I. I 5 per 100 patient-years. These risks persisted beyond three months of anticoagulation. The case fatality rate of major bleeding was $9.1 \%$ and the rate of intracranial bleeding was 0.65 per 100 patient-years for anticoagulation beyond three months. ${ }^{6}$

It has been estimated that 80 recurrent PEs and two to ten deaths per 1,000 patients can be prevented after one year by continuing anticoagulation beyond six months. However, there will be 20 to 60 major bleeds and two to six deaths with this approach. ${ }^{7}$ Thus, we can not recommend giving long-term anticoagulation treatment to all idiopathic VTE patients as benefits do not clearly outweigh risks.
With any long-term drug therapy, the most important issue is one of compliance. This is particularly important when the drug concerned can cause potentially lifethreatening adverse effects if taken in a higher than recommended dose. Many of the patients with VTE are older adults who are more likely to have haemorrhagic complications. ${ }^{8}$ Poor compliance may be an important issue with them. They are more sensitive to the effect of warfarin' and are likely to be on multiple drugs which may interact with warfarin. They are also more likely to bleed with anticoagulant therapy because of their underlying medical and surgical problems.

\section{COST AND INCONVENIENCE}

Taking oral anticoagulant therapy with warfarin and other vitamin $\mathrm{K}$ antagonists requires careful monitoring and regular INR checks. Patients living in remote areas may have to travel long distances at regular intervals for these tests. It can be argued that near-patient testing and the use of new oral anticoagulants which do not need routine monitoring can overcome these inconveniences. At present the primary care cost of anticoagulation per patient is estimated to be around $£ 322$ per year in the UK. ${ }^{10}$ With rational and evidence-based decision-making, this cost can potentially be avoided in a large number of patients with idiopathic VTE.

\section{CONCLUSIONS}

When planning the management of any medical condition, the important considerations are the safety and efficacy of the proposed treatment. 'First do no harm' is one of the key ethical principles. Evidence suggests that in patients with idiopathic VTE the risk of recurrent events is relatively low. Any benefit such patients may gain by anticoagulation beyond three months is negated by the risk of serious haemorrhages, which may even be fatal. Therefore, we believe that there is a need to develop methods to identify patients with idiopathic VTE who are at a high risk of developing recurrent events. Some have thrombophilia or ongoing risk factors such as cancer, which makes it easier to decide about long-term anticoagulation.

In patients who do not have such obvious indications, the decision can be more difficult and controversial. Attempts have been made by some studies to identify high-risk patients. A meta-analysis has shown that elevated D-dimer levels measured one month after discontinuation of anticoagulation in patients with idiopathic VTE identifies those who are at higher risk of recurrence." Another study has reported that, after adjustment for clinical risk factors, an elevated D-dimer level (between one and two months) after completion of six months' anticoagulation is significantly associated with an increased risk of unprovoked recurrent thrombosis (hazard ratio $2.0,95 \% \mathrm{Cl}$ I.0I-3.9). This study suggests a $10 \%$ annual risk of recurrence after 
stopping anticoagulant therapy in a male with an unprovoked first episode of VTE and a positive D-dimer. ${ }^{12}$ Such a patient may benefit from long-term anticoagulation. On the other hand, a female patient with provoked or unprovoked VTE and a negative D-dimer has around $2 \%$ annual risk of recurrence and, therefore, may not benefit from long-term anticoagulation. ${ }^{2}$ Further refinements in predicting recurrence will help us to make a rational choice of anticoagulant regime.

\section{REFERENCES}

I British Thoracic Society. Guidelines for the management of suspected acute pulmonary embolism. Thorax 2003; 58:470-84. doi:I0.II36/thorax.58.6.470

2 Kearon C, Kahn SR, Agnelli G et al. Antithrombotic therapy for venous thromboembolic disease: American College of Chest Physicians evidence-based clinical practice guidelines (8th edition). Chest 2008; 133;454S-545S. doi:10.1378/chest.08-0658

3 Campbell IA, Bentley DP, Prescott RJ et al. Anticoagulation for three versus six months in patients with deep vein thrombosis or pulmonary embolism, or both: randomised trial. BMJ 2007; 334:674-7. doi:I0.I I36/bmj.39098.583356.55

4 Douketis JD, Gu CS, Schulman S et al.The risk for fatal pulmonary embolism after discontinuing anticoagulant therapy for venous thromboembolism. Ann Intern Med 2007; 147:766-74.

5 Agnelli G, Prandoni P, Santamaria MG et al. Three months versus one year of oral anticoagulant therapy for idiopathic deep venous thrombosis. Warfarin Optimal Duration Italian Trial Investigators. N Engl J Med 200 I; 345: I 65-9. doi:I0.1056/NEJM200 I07/93450302

6 Linkins L-A, Choi PT, Douketis JD. Clinical impact of bleeding in patients taking oral anticoagulant therapy for venous thromboembolism: a meta-analysis. Ann Intern Med 2003; 139:893-900.

7 Bounameaux $\mathrm{H}$, Perrier A. Duration of anticoagulation therapy for venous thromboembolism. Hematology Am Soc Hematol Educ Program 2008:252-8. doi:I0.I I82/asheducation-2008.I.252
We have presented the evidence that the incidence of recurrent VTE is relatively low and the benefits of longer duration of anticoagulant therapy are clearly outweighed by the risk of serious, including fatal, bleeding. Should duration of anticoagulation be 'fixed and evidence-based, or flexible and personalised?' asks one author. ${ }^{13}$ We feel that, ideally, it should be both evidencebased and personalised.
8 Palareti G, Hirsh J, Legnani C et al. Oral anticoagulation treatment in the elderly: a nested, prospective, case-control study. Arch Intern Med 2000; 160:470-8. doi:10.1001/archinte.160.4.470

9 Routledge PA, Chapman PH, Davies DM et al. Factors affecting warfarin requirements. Eur J Clin Pharmacol 1979; 15:319-22. doi:I0.1007/BF00558434

I0 National Institute for Health and Clinical Excellence. Atrial fibrillation: the management of atrial fibrillation: costing report. London: NICE; 2006. Available from: http://www.nice.org.uk/nicemedia/pdf/ CG036costingreport.pdf

II Bruinstroop E, Klok FA, van de Ree MA et al. Elevated D-dimer levels predict recurrence in patients with idiopathic venous thromboembolism: a meta-analysis. J Thromb Haemost 2009; 7:6 I I-8. doi:I0. I III/j.1538-7836.2009.03293.x

12 Baglin T, Palmer C R, Luddington R et al. Unprovoked recurrent venous thrombosis: prediction by D-dimer and clinical risk factors. J Thromb Haemost 2008; 6:577-82. doi:I0.I I I I/j.I538-7836.2008.02889.x

13 Goldhaber SZ. Optimal duration of anticoagulation after venous thromboembolism: fixed and evidence-based, or flexible and personalized? Ann Intern Med 2009; 150:644-6.

\section{Anticoagulation for idiopathic venous thromboembolism should be given for longer than three months}

\section{TM Hyers}

Venous thromboembolism (VTE) is usually treated with 5-10 days of a heparin-related product, followed by oral anticoagulation with a vitamin $\mathrm{K}$ antagonist.' The duration of oral anticoagulation is often tailored to the perceived risk of recurrent VTE. Three months of anticoagulation is recommended for patients with a first VTE and transient or reversible risk factors such as trauma, surgery, immobilisation or oestrogen use. Prolonged or indefinite anticoagulation is recommended for patients with recurrent VTE, cancer or anti-phospholipid antibodies.

A first episode of idiopathic VTE, also known as primary or unprovoked VTE, is associated with a higher rate of recurrent VTE than that associated with provoked VTE (VTE associated with an identified risk factor). This difference in risk (two- to three-fold) is particularly notable when the identified risk factor is reversible or transient. The degree of increased risk with idiopathic disease is difficult to predict in individual cases, and often remains so even after an exhaustive and expensive search for a risk factor. Because of the increased risk associated with idiopathic VTE, many physicians believe that anticoagulation should be given for longer than three months. This approach immediately raises two questions: How long should anticoagulation be given to these patients? And how do we value the competing risks of recurrent VTE and haemorrhage when giving prolonged anticoagulation? The answer to the first question can be sought in the medical literature. The answer to the second question is ultimately a decision made by informed patients after weighing the competing risks and the personal burden of continuing prolonged anticoagulation.

Clinical trials that have examined the duration of anticoagulation have been admirably summarised in a recent exhaustive review, but the trials provide only 
partial answers.' In assessing these trials, several cautions are necessary. Many of the trials were not powered to detect small differences in bleeding rates, included not only patients with idiopathic disease but also those with known risk factors, had variable follow-up periods (up to one year) after anticoagulation was stopped and used suboptimal diagnostic criteria for recurrent VTE (the method of diagnosis was not always specified). However, the trials taken together indicate that prolonged anticoagulation does reduce the risk of recurrence after a first idiopathic VTE, albeit with possibly an increased bleeding rate. Shorter durations of treatment did not produce much difference. In comparison to three months of anticoagulation, six months seemed to offer little additional benefit. ${ }^{2,3}$

Twelve months of anticoagulation reduced the risk of recurrence during the period of anticoagulation. ${ }^{4}$ However, during extended follow-up after stopping 12 months of therapy, the rate of recurrence seemed to catch up to the rate seen with three months of therapy. ${ }^{4}$ Thus, 12 months of anticoagulation may only postpone a seemingly inevitable recurrence.

What about even longer periods of anticoagulation? This anticoagulation regimen has been called prolonged or indefinite, meaning there is no set length of treatment. These studies not only included patients with a first idiopathic VTE but also some patients with recurrent disease and patients with idiopathic disease whose serum D-dimer was elevated one month after stopping an initial course of therapy. ${ }^{5-8}$ The three trials with an anticoagulation target international normalised ratio (INR) of 2.5 (range $2.0-3.0$ ) showed a $90 \%$ risk reduction with prolonged treatment. The trial with an INR range of 1.5-2.0 showed a $60 \%$ risk reduction. ${ }^{8}$ The risk of serious bleeding varied between $\mathrm{I}-3 \%$ yearly, and more recent trials indicate that an annual serious bleeding rate of around $1 \%$ can be attained with standard intensity anticoagulation. ${ }^{8,9}$ It may be that any duration of treatment of idiopathic VTE only postpones a recurrence. However, the trials hint that recurrence rates of VTE begin to reach a plateau after two years of anticoagulation. ${ }^{5-8}$

The answer to the second question is a value judgement and requires the active participation of the patient. I think the authors of the recent review recognised this fact when they recommended three months of anticoagulation for patients with a first idiopathic event, followed by an evaluation of the risk-benefit ratio of longer-term therapy.'
They recommended longer-term therapy for those with low bleeding risk and when good anticoagulant monitoring is available. The authors acknowledge that their recommendation favoured the prevention of recurrent VTE over the burden of long-term anticoagulation. The informed patient must decide what are acceptable risks for both recurrent VTE and bleeding. In my experience, many patients are more concerned with bleeding risk and the burden of therapy than with recurrent disease, particularly after one or two years of treatment. In fact, patients in the studies of indefinite periods of anticoagulation usually stopped treatment after a serious haemorrhage or after deciding that continuing treatment with a vitamin $\mathrm{K}$ antagonist was too burdensome.

In my practice, after reviewing the risks and benefits of treatment of a first idiopathic VTE, I recommend at least one year of anticoagulation unless patients are at high bleeding risk or proper anticoagulant monitoring is unavailable. I make it clear that, in my opinion, optimum therapy should be for two years. However, I also stress that the decision must be collaborative and that a patient may stop treatment at any time when the burden of continued therapy is perceived to be too large.

Recently, regimens have been studied that use a flexible duration of anticoagulation that is tied to an objective study of residual thrombus or a laboratory test. Investigators used venous ultrasonography before stopping anticoagulation or serum D-dimer testing one month after stopping anticoagulation in an attempt to identify patients at higher risk of VTE recurrence., ${ }^{710}$ Patients with positive ultrasound findings or increased D-dimer levels were found to have higher recurrence rates if anticoagulation was stopped. The obvious inference from the studies is that a recommendation should be made for further anticoagulation if the test is positive. This approach looks promising, but these studies had relatively small numbers of patients. Confirmatory studies tailoring duration of therapy based on results of the test are needed before firm recommendations can be made.

The current debate may change in the near future when new oral anticoagulants that require no monitoring become available. Patients may perceive these drugs to be less burdensome than a vitamin $\mathrm{K}$ antagonist. However, new oral anticoagulants will be associated with bleeding, and patients and clinicians will still have to balance this risk with that of recurrent VTE.

\section{REFERENCES}

I Kearon C, Kahn SR, Agnelli G et al. Antithrombotic therapy for venous thromboembolic disease: American College of Chest Physicians evidence-based clinical practice guidelines (8th edition). Chest 2008; 133;454S-545S. doi:10.1378/chest.08-0658

2 Campbell IA, Bentley DP, Prescott RJ et al. Anticoagulation for three versus six months in patients with deep vein thrombosis or pulmonary embolism, or both: randomised trial. BMJ 2007; 334:674-7. doi:10.1 I36/bmj.39098.583356.55 
3 Pinede L. Ninet J. Duhaut $P$ et al. Comparison of 3 and 6 months of oral anticoagulant therapy after a first episode of proximal deep vein thrombosis or pulmonary embolism and comparison of 6 and 12 weeks of therapy after isolated calf deep vein thrombosis. Circulation 200I; 103:2453-60.

4 Agnelli G, Prandoni P, Santamaria MG et al. Three months versus one year of oral anticoagulant therapy for idiopathic deep venous thrombosis. Warfarin Optimal Duration Italian Trial Investigators. N Engl J Med 200I; 345:165-9. doi: I0.I056/NEJM200I07/93450302

5 Schulman S, Granqvist S, Holmstrom M et al. The duration of oral anticoagulant therapy after a second episode of venous thromboembolism. N Engl J Med 1997; 336:393-8. doi:10.1056/ NEJMI9970206336060I

6 Kearon C, Gent M, Hirsh J et al. A comparison of three months of anticoagulation with extended anticoagulation for a first episode of idiopathic venous thromboembolism. N Engl J Med 1999; 340:90I-7. doi:I0.1056/NEJMI9990325340 I 20 I
7 Palareti G, Cosmi B, Legnani C et al. D-dimer testing to determine the duration of anticoagulation therapy. $N$ Engl J Med 2006; 355: 1780-9. doi: I0.1056/NEJMoa054444

8 Ridker PM, Goldhaber SZ, Danielson E et al. Long-term lowintensity warfarin therapy for prevention of recurrent venous thromboembolism. N Engl J Med 2003; 348: 1425-34. doi:10.1056/ NEJMoa035029

9 Kearon C, Ginsberg JS, Kovacs MJ et al. Comparison of lowintensity warfarin therapy with conventional-intensity warfarin therapy for long-term prevention of recurrent venous thromboembolism. N Engl J Med 2003; 34:63I-9. doi:10.1056/ NEJMoa035422

10 Prandoni P, Prins MH, Lensing AW et al. Residual thrombosis on ultrasonography to guide the duration of anticoagulation in patients with deep venous thrombosis: a randomized trial. Ann Intern Med 2009; 150:577-85.

\section{EVENING MEDICAL UPDATES: SEPTEMBER 2010 - JUNE $20 \mathrm{II}$}

Open to all medical personnel. Evening Medical Updates start at $6.30 \mathrm{pm}$ and close at approximately $8.15 \mathrm{pm}$.

Fits/seizures

21 September 2010

Acute confusion 26 October 2010

Acute back pain 16 November 2010

Falls 14 December 2010

Jaundice 25 January 2011

Weakness/paralysis 22 February 2011

Palpitations 22 March 2011

Vomiting/Nausea 26 April 2011

Headache 24 May 20II

Haematemesis/melaena
Evening Medical Updates are also webstreamed live and video-linked to sites throughout the UK. Check online to see if there is a venue near you, or email a.fairbairn@rcpe.ac.uk if you are interested in live webstreaming or your hospital video-linking into the EMU programme of events.

For further information please contact: Mrs Anne Fairbairn, Education, Training \& Standards Department, Royal College of Physicians of Edinburgh, 9 Queen Street, Edinburgh EH2 IJQ Tel: 0I3I $2473649 \quad$ Fax:0I3I 2204393

E-mail: a.fairbairn@rcpe.ac.uk

\section{Full programme and details at} http://events.rcpe.ac.uk 